\title{
The clinical impact of gut microbiota in chronic kidney disease
}

\author{
So Mi Kim ${ }^{1}$ and Il Han Song ${ }^{2}$
}

Divisions of ${ }^{1} \mathrm{Nephrology}$ and ${ }^{2}$ Hepatology, Department of Internal Medicine, Dankook University Hospital, Cheonan, Korea

\section{Received : August 10, 2020 \\ Accepted: September 7, 2020}

\section{Correspondence to}

Il Han Song, M.D.

Division of Hepatology,

Department of Internal Medicine,

Dankook University Hospital, 201

Manghyang-ro, Dongnam-gu,

Cheonan 31116, Korea

Tel: +82-41-550-3924

Fax: +82-41-556-3256

E-mail: ihsong21@dankook.ac.kr

https://orcid.org/0000-0003-3975-

6342

This paper was contributed by The Korean Society of Nephrology.
Gut microorganisms play critical roles in both maintaining host homeostasis and the development of diverse diseases. Gut dysbiosis, an alteration of the composition and function of gut microorganisms, is commonly seen in patients with chronic kidney disease (CKD). CKD itself contributes to a disruption of the symbiotic relationship between the gut microbiota and the host, while the resulting gut dysbiosis may play a part in stage progression of CKD. This bidirectional relationship supports the concept that the gut microbiota is considered a novel focus for the pathogenesis and management of CKD. This article examines the interaction between the gut microbiota and the kidney, the mutual effects of dysbiosis and CKD, and possible treatment options to restore gut eubiosis, and reduce CKD progression and its related complications.

Keywords: Renal insufficiency, chronic; Gastrointestinal microbiome; Dysbiosis; Uremic toxins

\section{INTRODUCTION}

Chronic kidney disease (CKD) is a global health threat with a prevalence of $8 \%$ to $16 \%$ worldwide, and has been gradually increasing in recent decades due to the aging population, the increase in chronic debilitating diseases, and decreased mortality of patients at risk for renal impairment [1]. CKD is mainly caused by diabetes mellitus (DM), hypertension (HTN), and glomerulonephritis, eventually progresses to end-stage renal disease (ESRD) and is associated with high morbidity and mortality related to cardiovascular diseases, despite active medical treatment [1-3]. Regardless of attempts to attenuate the progression of CKD, there have been no significant breakthroughs in the management of patients with CKD for decades, due to a lack of understanding of the pathogenesis and pathophysiology of CKD. Recently, as interest in the gut microenvironment has increased, the gut microbiota is seen as a key modulator of human health, to the extent that it has been proposed to be an essential organ in the human body $[4,5]$. The relationship between the gut microbiota and the host organs has attracted great attention in both the maintenance of host homeostasis and the development of diverse diseases [4-7]. CKD contributes to the alteration of composition and function of the gut microbiota, called dysbiosis. In contrast, there is growing evidence that dysbiosis itself contributes to CKD development and progression via several mechanisms such as microbiota-derived toxins, immune-mediated products and neuroendocrine-mediated substances [6-10]. This bidirectional relationship supports the concept that the gut microbiota is consid- 
ered a novel focus for the cause and treatment of CKD. This review will explore the interface between the gut microbiota and kidney, address the clinical effects of gut microbiota on CKD onset and progression, and include the results of clinical trials for possible treatment options in patients with CKD.

\section{SYMBIOSIS: GUT MICROBIOTA AND HEALTHY SUBJECTS}

The gut microbiota is defined as all the living gut microorganisms that form a symbiotic relationship with the host, and includes mainly bacteria, but also viruses, archaea, fungi, and unicellular eukaryotes $[4,5]$. The human gut contains approximately 1 trillion microorganisms with thousands of species encoding more than 3 million genes (150-fold more than the human genome) [4]. In healthy subjects, the microbiota in the colon is mainly composed of five phyla; significant proportions are Firmicutes and Bacteroidetes (collectively 90\%), followed by Actinobacteria, Verrucomicrobia, and, in a lower proportion, Proteobacteria $[4,5,10]$. The microbiota plays a fundamental role in the breakdown of indigestible plant polysaccharides from the diet, biosynthesis of short-chain free fatty acids (SCFAs) and vitamins, biotransformation of conjugated bile acids and degradation of dietary oxalates, induction and training of our immune system, and reducing allergic responses to foods and/or environmental antigens [4-8]. The colonization of microbiota in the gut begins immediately after birth and reaches a mature composition by the age of 3 years. Thereafter, it is maintained throughout adulthood. However, the pattern of microbiota including its richness, diversity, and uniformity, is influenced by age, hygiene, infection, exposure to allergens, drugs, and several diseases $[9,10]$. Therefore, an imbalance in composition of the gut microbiota may be related to disease development.

\section{THE EFFECT OF CKD ON GUT MICROBIOTA}

CKD is characterized by the accumulation of uremic toxins, and is commonly associated with disturbed mechanical changes in the gastrointestinal tract such as edema of the intestinal wall and alteration of colonic transit $[8,9,11]$. Patients with CKD often experience dietary restrictions and the frequent consumption of iron, phosphorus binding agents, and antibiotics [8-12]. These characteristics may induce the development of dysbiosis, and furthermore may inversely promote disease progression to advanced stages of CKD. Fig. 1 shows a reciprocal interaction between gut dysbiosis and CKD.

\section{The effect of uremic toxins from CKD on dysbiosis}

As renal function decreases, waste products called uremic toxins accumulate in the blood, and also increase in the intestinal epithelium $[8,9,13]$. This change in the gut promotes the colonization of bacteria that can use urea as an energy source by producing the enzymes urease or uricase. Urease is a cytosolic enzyme that catalyzes the hydrolysis of urea to ammonia and carbon dioxide. Consequently, the markedly increased formation of ammonia increases the gut $\mathrm{pH}$, mediates enterocolitis by the breakdown of gut epithelial tight junctions, and facilitates endotoxemia and systemic inflammation [7-9,13-16]. The uric acid originating from purine metabolism is mainly excreted via the kidney; however as renal function decreases, the colon replaces the kidney as the primary site of uric acid excretion $[9,15,16]$. The high concentration of uric acid increases the bacteria containing urease and uricase and influences changes in the gut microenvironment via the same pathway. Several studies have demonstrated that CKD patients have significant changes in the gut microbiota with loss of $\alpha$-diversity, $\beta$-diversity, and richness [8-10]. In a study of 24 stable ESRD patients, Wong et al. [17] also showed reduction in the Lactobacillaceae and Prevotellaceae families, which can express the butyrate kinase gene involved in protective processes, and confirmed that most of the microbiota has urease, uricase or tryptophanase activity; 12 of 19 bacteria families have urease activity (including Alteromonadaceae, Cellulomonadaceae, Clostridiaceae), five have uricase activity (Cellulomonadaceae, Dermabacteraceae, Micrococcaceae, Polyangiaceae, and Xanthomonadaceae), and three have tryptophanase activity (Clostridiaceae, Enterobacteriaceae, and Verrucomicrobiaceae). This alteration of gut microbiota can promote the acceleration of dysbiosis and its metabolic activities, and lead to the change of gut structure called leaky gut syndrome. Vaziri et al. [16] reported a marked reduction in the amount of tight 


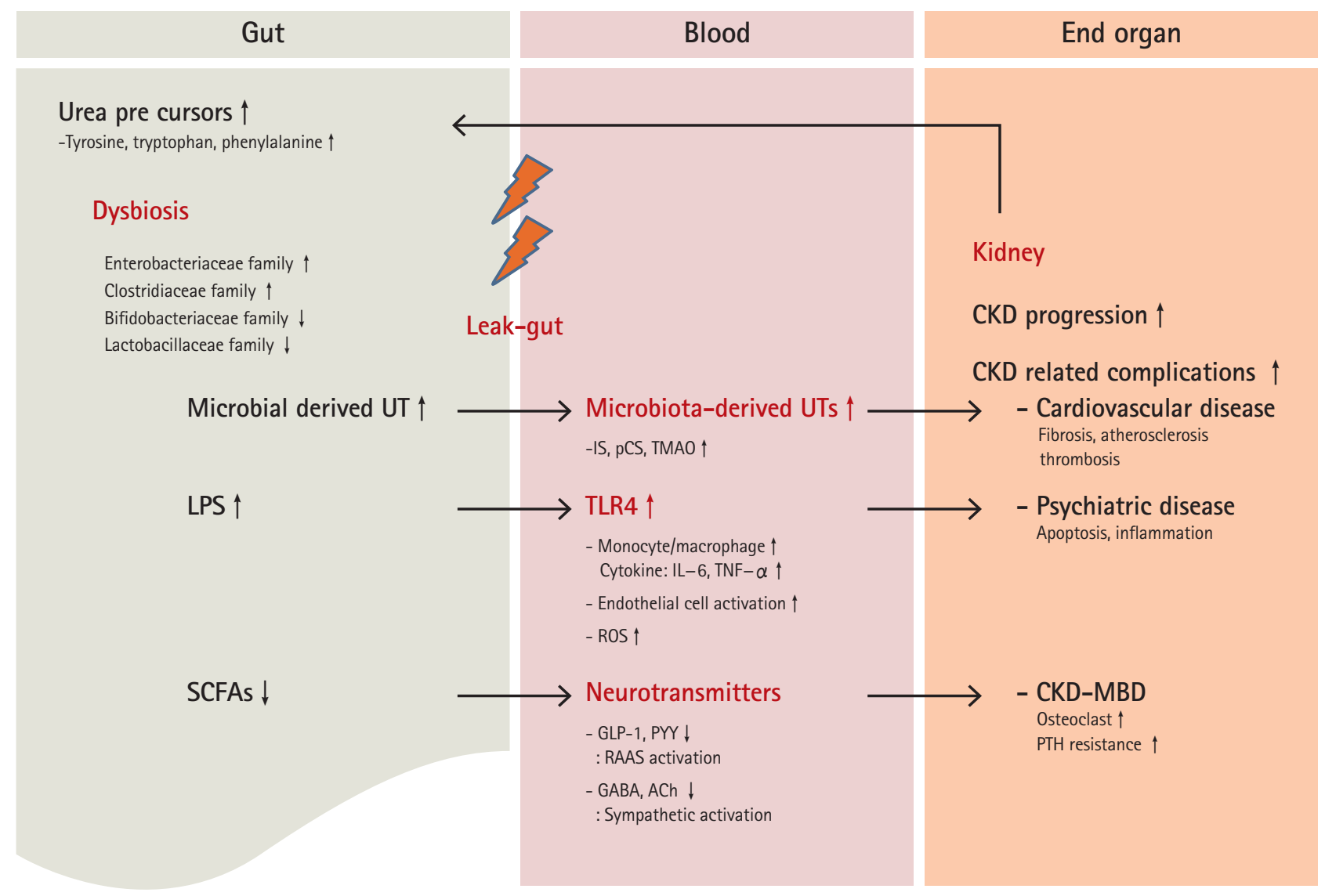

Figure 1. The reciprocal interaction between gut dysbiosis and CKD. UT, uremic toxin; LPS, lipopolysaccharide; SCFA, shortchain free fatty acid; IS, indoxyl sulfate; pCS, p-cresyl sulfate; TMAO, trimethylamine N-oxide; TLR4, toll-like receptor-4; IL6 , interleukin-6; TNF- $\alpha$, tumor necrosis factor- $\alpha$; ROS, reactive oxygen species; GLP-1, glucagon-like peptide 1 ; PYY, peptide YY; RAAS, renin-angiotensin-aldosterone system; GABA, gamma aminobutyric acid; ACh, acetylcholine; CKD, chronic kidney disease; MBD, mineral bone disorder; $\mathrm{PTH}$, parathyroid hormone.

junction-related proteins such as claudin-1, occludin, and zonula occludens 1 in the colonic mucosa of animals with CKD. In addition, Farhadi et al. [18] showed several histological changes such as reduction of villous height, elongation of the crypts, and infiltration of the lamina propria with inflammatory cells in the intestines of CKD patients. These pathological changes in the gut suggest that translocation of bacteria and the influx of endotoxins across the intestinal wall can contribute to inducing systemic inflammation in CKD.

\section{Other CKD-related factors that contribute to dysbiosis}

The classic CKD diet is generally composed of light meals containing low concentrations of sodium, potassium and phosphate, to reduce their adverse metabolic effects [19]. This type of diet limits the consumption of potassium-rich foods such as fruits, vegetables, nuts, and high-fiber products. Indigestible carbohydrates are not only essential nutrients for the metabolism of gut microbiota but also precursors of short-chain fatty acids (SCFAs) related to immune regulation, glucose and lipid metabolism, gut integrity, and appetite $[4,19,20]$. Therefore, the CKD diet contributes to a decrease in the production of SCFAs and increases the amino nitrogen load, which can transform into uremic toxins $[8,20]$. CKD patients are commonly prescribed multiple drugs including iron-containing compounds, phosphate binders, and antibiotics $[20,21]$. This long-term and high pill burden for CKD patients can extensively influence the composition and richness of the gut microbiota. 


\section{THE EFFECT OF DYSBIOSIS ON CKD PROGRESSION}

CKD-induced dysbiosis can contribute to CKD progression and the development of CKD-related adverse events through diverse mechanisms such as microbiota-derived metabolites, a disrupted intestinal barrier, and changes in the neuroendocrine immune system.

\section{The effect of microbiota-derived toxins on CKD}

Uremic toxins can be classified based on their site of origin: endogenous (mammalian metabolism), exogenous (diet), or microbial [22,23]. Among these original site-specific toxins, three molecules including indoxyl sulfate (IS), p-cresyl sulfate (pCS), and trimethylamine $\mathrm{N}$-oxide (TMAO), are microbiota-derived uremic toxins involved in the development of complications including cardiovascular disease and mortality as well as the pathogenesis and progression of CKD. IS, a protein-binding uremic toxin and one of the microbiota-derived metabolites, is synthesized from dietary tryptophan by bacterial fermentation, and is excreted in the urine. IS converted from indole in the liver contributes to peripheral vascular disease and thrombosis of vascular access [2224]. Its serum concentration increases in proportion to a decrease in renal function. In a prospective study of 268 CKD patients [24], Wu et al. [24] reported that the baseline concentration of IS can be a predictor of loss of renal function. Some experimental studies have demonstrated that IS mediates the renal expression of genes related to tubulointerstitial fibrosis, such as transforming growth factor $\beta 1$ and a tissue inhibitor of metalloproteinases $[23,25]$. Another experimental study showed that mouse podocytes exposed to IS for 8 weeks exhibited a pro-inflammatory phenotype, perturbed actin cytoskeleton, decreased expression of podocyte-specific genes, and decreased cell viability [26]. pCS is a colonic fermentation product of tyrosine and phenylalanine catabolism by anaerobic gut bacteria. Once p-cresol is absorbed, it is conjugated with other substances in the liver, where a sulfate group is added [6-8]. In a CKD rat model, pCS increased the production of reactive oxygen species (ROS), activating nicotinamide adenine dinucleotide phosphate oxidase and increasing caspase-3 activity, leading to an increased apoptosis ratio [27]. In another experimental study of half-nephrectomized mice, IS or pCS activated the intrarenal renin-angiotensin-aldosterone system (RAAS) and induced interstitial fibrosis and glomerulosclerosis [28]. TMAO is synthesized from dietary choline, phosphatidylcholine, and L-carnitine. Pelletier et al. [29] reported that the serum TMAO concentration negatively correlates with the glomerular filtration rate (GFR) in patients with CKD. Tang et al. [30] showed that an elevated level of TMAO was associated with the degree of tubulointerstitial fibrosis in an animal model and poorer overall survival in CKD patients. Stubbs et al. [31] also reported that TMAO increases the phosphorylation of $\mathrm{SMAD}_{3}$, an important regulator of fibrosis, and consequently enhances atherosclerosis and thrombosis, leading to an increased incidence of coronary artery disease. Therefore, TMAO has been proposed as a potential surrogate marker to detect early cardiovascular risk in patients with CKD. These accumulating data suggest that increased microbiota-derived metabolites are definite risk factors that contribute to CKD development and progression, and attempting to reduce microbiota-derived uremic toxins seems to be a reasonable therapeutic strategy for CKD patients.

\section{The immunological effects of dysbiosis on CKD}

The gut microbiota can provide a large number of potential immunostimulatory bacterial products. Recently, this has been considered a critical exogenous trigger of host immune dysfunction $[4,5]$. The leaky gut in CKD patients can facilitate the influx of microbiota-derived products into the hepatic portal system and systemic circulation across the intestine wall. A lipopolysaccharide (LPS) is derived from the cell wall component of gram-negative bacteria [32-34]. LPS induces systemic inflammation via a cascade of inflammatory responses. Lipid A is a component of LPS and serves as the microbe-specific molecular signal that binds to the surface receptor complexes of immune cells (monocytes/macrophages), which comprise toll-like receptor-4 (TLR4) and myeloid differentiation (MD) factor-2. The formation of the TLR4-MD2-LPS complex activates the signaling pathway controlling the expression of inflammatory genes, leading to excessive production of pro-inflammatory cytokines such as interleukin-1 $\beta$ (IL-1 $\beta$ ), IL-6, and tumor necrosis factor $\alpha$ [33-35]. TLR4 is also highly expressed in the endothelial cell, vascular smooth muscle cell and adventitial fibroblast. Activated TLR4 promotes 
the recruitment of monocytes, transformation of macrophages to foam cells, and the expression of adhesion molecules, ROS, and pro-coagulant activity by intracellular inflammatory pathways mediated through nuclear factor- $\mathrm{\kappa B}$ and mitogen-activated protein (MAP) kinases [32-35]. Ultimately, these processes can initiate and promote atherosclerosis. McIntyre et al. [36] demonstrated that circulating bacterial endotoxin/LPS levels are increased in all stages of CKD and reach the maximum in dialysis patients, and suggested that systemic inflammation is also a strong and independent predictor of mortality in CKD. Szeto et al. [32] also showed that circulating endotoxin in patients undergoing peritoneal dialysis is related to systemic inflammation and features of atherosclerosis.

\section{The neuroendocrine effects of dysbiosis on CKD}

The gut is the second-most innervated organ in the body, and the gut microbiota communicates with the nervous system by producing several hormones and neurotransmitters $[4,7]$. The central and peripheral nervous systems regulate the circulatory system by modulating the sympathetic nervous system, RAAS, and pituitary hormone release. Jazani et al. [37] demonstrated that the gut microbiota activates the hypothalamic-pituitary-adrenal (HPA) axis and increases the secretion of serotonin and other neurotransmitters. Onal et al. [38] reported that Bifidobacteriaceae, Lactobacillaceae, and Prevotellaceae species can synthesize neurotransmitters such as $\gamma$-aminobutyric acid (GABA) and acetylcholine (ACh) and can promote production of intestinal incretin, glucagon-like peptide-1 and -2 (GLP-1, -2), and the gut hormone peptide YY. GABA is known to stimulate natriuresis and to suppress renal sympathetic nerve activity $[9,38]$. ACh and GLP-1 have been reported to increase the GFR by renal vasodilation and reducing angiotensin II [7,9,37]. Therefore, gut dysbiosis with reduction of bacteria species such as Bifidobacteriaceae and Lactobacillaceae in CKD causes activation of the RAAS and augmentation of sympathetic outflow and, ultimately, leads to HTN and CKD progression. In addition, changes in GLP and peptide YY can influence energy homeostasis by reduced energy expenditure, lipolysis, and insulin sensitivity and secretion, leading to obesity, hypercholesterolemia, insulin resistance, and/or DM. These are important risk factors for CKD development and can also contribute to CKD progression $[4,33,38]$.

\section{The clinical impact of dysbiosis on CKD-related complications}

Increasing evidence supports the concept that dysbiosis in CKD patients is associated with CKD-related complications such as HTN, cardiovascular events, miner$\mathrm{al}$ and bone disorder (MBD), and cognitive dysfunction [33,38]. The shift towards a predominantly proteolytic fermentation pattern in CKD promotes the increase of microbiota-derived toxins and increases systemic inflammation.

\section{Cardiovascular disease}

Cardiovascular disease is a main cause of morbidity and mortality in CKD patients. Beyond the gut-kidney axis, several reports suggest that diverse mechanisms including enhanced ROS production, leukocyte activation, pro-inflammatory cytokine production, myocyte hypertrophy, and RAAS activation contribute to the development and progression of cardiovascular disease, calling these relationships between the gut and heart the gutheart axis [7,38-41]. Lin et al. [42] showed that elevated levels of pCS and IS are associated with increased mortality in CKD patients while pCS, but not IS, is associated with an increased risk of cardiovascular events. A recent meta-analysis studying TMAO in 19,256 patients also demonstrated that elevated levels of TMAO and its precursors were associated with a 1.7-fold increased risk of major acute cardiovascular events and all-cause mortality in comparison with low TMAO [43].

\section{Cognitive dysfunction}

Psychiatric disease is common in CKD patients and is associated with increased morbidity and mortality $[9,39,44,45]$. Although estimates vary, studies have shown that combined depression and cognitive dysfunction is present in approximately $20 \%$ to $25 \%$ of CKD patients [7-9,44,45]. Growing evidence suggests that gut dysbiosis promotes dysregulation of the HPA axis with overproduction of glucocorticoids, alterations in levels of neurotransmitters, and activation of pro-inflammatory cytokines, called the gut-brain axis [39,45-48]. Gut microbiota-derived toxins play an important role in the pathogenesis of cognitive dysfunction by direct toxicity or other putative factors such as oxidative stress, inflam- 
mation, endothelial dysfunction and vascular calcification $[7,45,46]$. IS crosses the blood-brain barrier through the organic anion transporter-3 (OAT-3), and may accumulate in the brain in CKD due to dysfunctional OAT-3 [46]. Elevated IS induces inflammation and apoptosis of human astrocytes and glial cells through oxidative stress induction and MAP kinase pathway inhibition [45-48]. Aryl hydrocarbon receptor (AhR), known as a ligand-activated transcriptional factor, is mainly expressed in the hippocampus, cerebral cortex, and cerebellum, and has been implicated in sensorimotor and cognitive functions [7,9,47,48]. Elevated IS activates AhR in astrocytes, which is likely to promote further oxidative stress, leading to sensorimotor and cognitive dysfunction in CKD [47,48]. In a clinical study with 260 hemodialysis (HD) patients, Lin et al. [47] showed that the circulating free form of IS is significantly associated with lower cognitive function test scores, particularly in cognitive abilities screening instrument domains of memory, mental manipulation, and language ability. Further clinical studies based on molecular regulation in the expression of uremic toxins are needed in CKD patients.

\section{CKD-MBD}

CKD-MBD is a syndrome recently re-named to embody the biochemical, skeletal, and cardiovascular pathophysiology, not limited to bone disease [49]. CKD-MBD is associated with poor outcomes in terms of myocardial infarction, stroke, bone fracture, and all-cause mortality [50]. Growing evidence indicates elevated uremic toxins are involved in the development of bone abnormalities in CKD [51]. Mozar et al. [50] demonstrated that IS in- hibited both osteoclast differentiation and bone-resorbing activity in monocyte/macrophage cellular models. Nii-kono et al. [52] also found that IS suppressed parathyroid hormone (PTH)-stimulated intracellular cyclic adenosine monophosphate production and decreased PTH receptor expression in a primary osteoblast culture from mouse calvaria. In a clinical study with $47 \mathrm{HD}$ patients, Goto et al. [53] showed that IS correlated negatively with alkaline phosphatase, particularly bone-specific alkaline phosphatase, regardless of intact PTH activity. These findings strongly suggested that IS not only inhibits osteoblast function but also has an inhibitory effect on osteoclasts and PTH and, thus, could affect bone remodeling in CKD patients.

\section{TARGETED TREATMENTS IN THE CONTEXT OF GUT MICROBIOTA}

Recent evidence about the pathogenic consequences of gut dysbiosis on CKD supports the concept that restoring symbiosis in the gut could be an effective and beneficial targeted treatment for CKD. This would have the additional significance of being more physiological in that it is related to basic diet and digestion. In preclinical or clinical investigations potential therapeutic options have focused on diet modification with healthy supplements, oral adsorbents preventing gut absorption of uremic toxins, modification of the microbiota composition, and modulation of influx/efflux renal transporters for uremic toxins (Table 1). Of these, the treatments currently most commonly used in clinical

Table 1. Potential therapeutic interventions on the aberrant axis of gut microbiota and chronic kidney disease

\begin{tabular}{ll}
\hline Diet modification & Low protein diet (decrease dietary uremic toxin precursors) \\
& High fiber diet (increase dietary renoprotective precursors, e.g., SCFAs) \\
Modulation of gut microbiota & $\begin{array}{l}\text { Prebiotics/probiotics/synbiotics } \\
\text { Fecal transplantation }\end{array}$ \\
\hline \multirow{2}{*}{ Blocking LPS and inflammation } & $\begin{array}{l}\text { Synthetic TLR4 antagonist } \\
\text { Synthetic lipid A analogue }\end{array}$ \\
\hline Adsorption of uremic toxins & Oral adsorbents (e.g., AST-120, sevelamer) \\
& $\begin{array}{l}\text { Carbon-based matrix based dialyzer } \\
\text { Plasma-binding protein infusion (usually albumin), ibuprofen on dialysis }\end{array}$ \\
\hline Modulation of renal transporters & Meclofenamate (increase the expression of OAT-1, OAT-3 in proximal tubules) \\
\hline
\end{tabular}

SCFA, short-chain fatty acid; LPS, lipopolysaccharid; TLR4, toll-like receptor-4; OAT, organic anion transporter. 
practice are described below.

\section{High fiber diets}

Resistant starch (RS) is a type of carbohydrate that is incompletely digested by human pancreatic amylases. High amylose maize-resistant starch type 2 (HAMRS2), a kind of RS found in starchy foods such as potato, corn, and banana, reaches the large intestine and serves as an energy source for beneficial bacteria such as Bifidobacterium and Lactobacillus [54,55]. Several studies have demonstrated the beneficial effects of HAMRS2 on CKD progression, decreased microbial diversity, and an increased Bacteroidetes-to-Firmicutes ratio in HAMRS2fed rats [54-56]. Vaziri et al. [56] also reported a favorable effect of this type of RS on reducing oxidative stress and inflammation, and restoring intestinal epithelial tight junctions in a CKD rat model. Krishnamurthy et al. [57] revealed that high fiber diets showed low inflammation and decreased all-cause mortality in a study analyzing data from 14,543 participants in the National Health and Nutrition Examination Survey III. Despite these potential renoprotective effects, high fiber diets have a limitation in alimentotherapy for patients with advanced stages of CKD due to their potassium and phosphorus content. Therefore, practical cooking techniques and counselling should be provided for safe intake in CKD patients.

\section{Prebiotics, probiotics, and synbiotics}

Prebiotics are nondigestible food ingredients that can help stimulate the growth of the selected or a limited number of bacteria in the colon $[58,59]$. Inulin, fructo-oligosaccharides, galacto-oligosaccharides, soya-oligosaccharides, xylo-oligosaccharides, and pyrodextrins are commonly used as prebiotics. They promote the growth of Bifidobacteria and Lactobacilli species, while suppressing other groups of bacteria such as Bacteroides, Clostridia, and Enterobacteria species [59]. Meijers et al. [6o] also demonstrated that serum concentrations of IS and pCS were significantly reduced by the oral intake of oligofructose-enriched inulin in $22 \mathrm{HD}$ patients. The prebiotic lactulose also improved kidney function by modifying gut microbiota and inhibiting the production of uremic toxins in 10-week-old adenine-induced CKD Wistar/ST male rats. In addition, lactulose decreased serum levels of creatinine and blood urea nitrogen (BUN) and ameliorated CKD progression by suppressing tubulointerstitial fibrosis [61]. Probiotics are defined as live microorganisms that confer a health benefit on the host when administered in adequate amounts [62]. Probiotics commonly consist of living bacteria such as Bifidobacteria species, Lactobacilli and Streptococci [63]. A pilot clinical trial in patients with CKD stages 3 and 4 showed significantly decreased BUN and improved quality of life after treatment with the Renadyl (Kibow Biotech Inc., Newtown Square, PA, USA) formulation of Lactobacillus acidophilus, Streptococcus thermophilus, and Bifidobacterium longum over 6 months [63]. However, the follow-up randomized controlled trial in 22 patients failed to show decreased uremic toxins or the sensation of enhanced well-being [64]. Some of the benefits of probiotics could be explained by persistent uremia-induced alterations in the gut biochemical milieu as well as by dietary or medicinal regimens that produce an unfavorable microenvironment for the symbiotic microbiota. Synbiotics are the combination regimen of prebiotics and probiotics. A randomized clinical trial by Guida et al. [65] showed a suppressive effect of short-term synbiotic treatment on plasma p-cresol levels without improvement of gastrointestinal symptoms in $30 \mathrm{CKD}$ patients after 4 weeks. A recent multicenter study in $42 \mathrm{HD}$ patients showed an improvement of gastrointestinal symptoms and decreased C-reactive protein after 2 months of treatment [66]. The recent randomized controlled trial of the effects of pre/pro/synbiotics is summarized in Table 2 $[63,67-76]$.

\section{Fecal microbiota transplantation}

Fecal microbiota transplantation (FMT), also known as a stool transplant, is another option to modify the gut microbiota $[77,78]$. However, there are few data about FMT for the treatment of CKD, except the use of FMT for the treatment of Clostridium difficile infection, which is a common complication in patients with CKD undergoing $\mathrm{HD}[77,78]$. Further research is needed to understand how FMT affects the progression of CKD.

\section{Adsorption therapy}

AST-120, an oral charcoal adsorbent, is widely used as a therapeutic agent in CKD patients to absorb circulating uremic toxins and precursors such as IS. Several animal studies have shown that, in addition to the change in 
Table 2. Randomized clinical trials of prebiotics, probiotics and synbiotics in CKD

\begin{tabular}{|c|c|c|c|c|}
\hline & Subject & Study deign & Dose of drug & Outcomes \\
\hline \multicolumn{5}{|l|}{ Prebiotics } \\
\hline $\begin{array}{l}\text { Poesen et al. } \\
\text { [68] }\end{array}$ & $\begin{array}{l}\text { ND CKD } \\
(\mathrm{n}=40)\end{array}$ & $\begin{array}{l}\text { Randomized, double-blind, } \\
\text { placebo-controlled }\end{array}$ & $\begin{array}{l}\text { Arabinoxylan oligosaccharides } 10 \mathrm{~g} \text { twice } \\
\text { daily for } 4 \text { weeks }\end{array}$ & TMAO $\downarrow$ \\
\hline $\begin{array}{l}\text { Ramos et al. } \\
{[69]}\end{array}$ & $\begin{array}{l}\text { ND CKD } \\
(\mathrm{n}=50)\end{array}$ & $\begin{array}{l}\text { Randomized, double-blind } \\
\text { placebo-controlled }\end{array}$ & $\begin{array}{l}\text { Fructooligosaccharide } 12 \text { g/day for } 3 \\
\text { months }\end{array}$ & $\begin{array}{l}\text { Total/free pCS } \downarrow \\
\text { independent of } \\
\text { eGFR }\end{array}$ \\
\hline $\begin{array}{l}\text { Esgalhado } \\
\text { et al. [67] }\end{array}$ & $\begin{array}{l}\mathrm{HD} \\
(\mathrm{n}=31)\end{array}$ & $\begin{array}{l}\text { Randomized, double blind, } \\
\text { placebo-controlled }\end{array}$ & $\begin{array}{l}\text { Hi-Maize }{ }^{\circledR} 260 \text { powder (which contains } 16 \\
\text { g of RS) } 26 \text { g/day for } 4 \text { weeks }\end{array}$ & $\begin{array}{l}\text { IL-6 } \\
\text { IS } \downarrow \\
\text { TBARS } \downarrow\end{array}$ \\
\hline $\begin{array}{l}\text { Laffin et al. } \\
{[70]}\end{array}$ & $\begin{array}{l}\mathrm{HD} \\
(\mathrm{n}=20)\end{array}$ & $\begin{array}{l}\text { Randomized, double-blind, } \\
\text { placebo-controlled }\end{array}$ & $\begin{array}{l}\text { HAM-RS2, } 20 \mathrm{~g} / \text { day during the } 1 \text { st month, } \\
25 \mathrm{~g} / \text { day during the } 2 \text { nd month }\end{array}$ & $\begin{array}{l}\text { BUN } \downarrow \\
\text { IL-6, TNF- } \alpha \downarrow \\
\text { Faecalibacterium } \uparrow\end{array}$ \\
\hline \multicolumn{5}{|l|}{ Probiotics } \\
\hline $\begin{array}{l}\text { Ranganathan } \\
\text { et al. [63] }\end{array}$ & $\begin{array}{l}\text { CKD with } \\
\text { stage } 3,4 \\
(\mathrm{n}=13)\end{array}$ & $\begin{array}{l}\text { Randomized, } \\
\text { double-blind, } \\
\text { placebo-controlled }\end{array}$ & $\begin{array}{l}\text { Kibow biotics (Lactobacillus acidophilus } \\
\text { KB31, Streptococcus thermophilus KB27, } \\
\text { Bifidobacterium longum KB35) } \\
\left(\text { each } 1.5 \times 10^{10} \mathrm{CFU}\right) \\
\text { Total dose: } 9 \times 10^{10}(2 \text { capsule } \times 3 \text { ) for } 6 \\
\text { months }\end{array}$ & $\begin{array}{l}\text { BUN } \downarrow, \mathrm{Cr} \downarrow \\
\text { No change of } \\
\text {-GI symptom } \\
\text {-quality of life }\end{array}$ \\
\hline $\begin{array}{l}\text { Mafi et al. } \\
{[72]}\end{array}$ & $\begin{array}{l}\text { ND CKD } \\
(\mathrm{DM}) \\
(\mathrm{n}=60)\end{array}$ & $\begin{array}{l}\text { Randomized, } \\
\text { placebo-controlled }\end{array}$ & $\begin{array}{l}\text { Lactobacillus acidophilus ZT-L1, Bifidobacteri- } \\
\text { um bifidum ZT-B1, Lactobacillus reuteri ZT- } \\
\text { Lre, Lactobacillus fermentum ZT-L3 (each } 2 \times \\
10^{9} \text { ) } \\
\text { Total dose: } 8 \times 10^{9} \mathrm{CFU} / \text { day, for } 12 \text { weeks }\end{array}$ & $\begin{array}{l}\text { Fasting glucose } \downarrow \\
\text { Serum insulin } \uparrow \\
\text { HOMA-IR } \downarrow \\
\text { CRP } \downarrow \\
\text { HDL cholesterol } \uparrow \\
\text { Total glutathione } \uparrow \\
\text { Plasma betaine } \uparrow\end{array}$ \\
\hline $\begin{array}{l}\text { Eidi et al. } \\
{[71]}\end{array}$ & $\begin{array}{l}\mathrm{HD} \\
(\mathrm{n}=42)\end{array}$ & $\begin{array}{l}\text { Randomized, } \\
\text { triple-blind, } \\
\text { placebo-controlled }\end{array}$ & $\begin{array}{l}\text { Lactobacillus rhamnosus } 1.6 \times 10^{7} \mathrm{CFU} / \text { day } \\
\text { (1 capsule) for } 4 \text { weeks }\end{array}$ & $\begin{array}{l}\text { p-cresol and } \\
\text { phenol } \downarrow\end{array}$ \\
\hline $\begin{array}{l}\text { Borges et al. } \\
\text { [73] }\end{array}$ & $\begin{array}{l}\mathrm{HD} \\
(\mathrm{n}=46)\end{array}$ & $\begin{array}{l}\text { Randomized, } \\
\text { double-blind, } \\
\text { placebo-controlled }\end{array}$ & $\begin{array}{l}\text { Streptococcus thermophiles (KB19), } \\
\text { Lactobacillus acidophilus (KB27), } \\
\text { Bifidobacteria longum (KB31) } \\
\text { Totaling } 9 \times 10^{13} \mathrm{CFU} / \text { day (3 capsule) } \\
\text { for } 3 \text { months }\end{array}$ & \\
\hline \multicolumn{5}{|l|}{ Synbiotics } \\
\hline $\begin{array}{l}\text { Rossi et al. } \\
\text { [74] }\end{array}$ & $\begin{array}{l}\text { ND CKD } \\
(\mathrm{n}=31)\end{array}$ & $\begin{array}{l}\text { Randomized, } \\
\text { double-blind, } \\
\text { placebo-controlled }\end{array}$ & $\begin{array}{l}\text { Prebiotic: inulin, fructo-oligosaccharides, } \\
\text { and galacto-oligosaccharides } \\
\text { Probiotic: Lactobacillus, Bifidobacteria, and } \\
\text { Streptococcus genera } \\
\text { First } 3 \text { weeks: prebiotic, } 7.5 \mathrm{~g} \text {, and probiotic } \\
45 \text { billion CFU } \\
\text { Second } 3 \text { weeks: twice the dose for } 6 \text { weeks } \\
\text { with a dose escalation }\end{array}$ & $\mathrm{pCS} \downarrow$ \\
\hline $\begin{array}{l}\text { Dehghani } \\
\text { et al. }[76]\end{array}$ & $\begin{array}{l}\text { CKD with } \\
\text { stage } 3,4\end{array}$ & $\begin{array}{l}\text { Randomized, placebo-con- } \\
\text { trolled }\end{array}$ & $\begin{array}{l}\text { Synbiotic supplement 1,000 mg/day for } 6 \\
\text { weeks }\end{array}$ & $\mathrm{BUN} \downarrow$ \\
\hline $\begin{array}{l}\text { Guida et al. } \\
\text { [75] }\end{array}$ & $\mathrm{KT}(\mathrm{n}=36)$ & $\begin{array}{l}\text { Randomized, double-blind, } \\
\text { placebo-controlled }\end{array}$ & $\begin{array}{l}\text { Synbiotics (Probinul Neutro, CadiGroup, } \\
\text { Rome, Italy) } 5 \text { g powder } \\
\text { Total } 15 \mathrm{~g} / \text { day for } 15 \text { or } 30 \text { days }\end{array}$ & $\mathrm{pCS} \downarrow$ \\
\hline
\end{tabular}

CKD, chronic kidney disease; ND, non-dialysis; TMAO, trimethylamine N-oxide; pCS, p-cresyl sulfate; eGFR, estimated glomerular filtration rate; HD, hemodialysis; RS, resistant starch; IL-6, interleukin-6; IS, indoxyl sulfate; TBARS, thiobarbituric acid reactive substance; BUN, blood urea nitrogen; TNF- $\alpha$, tumor necrosis factor $\alpha$; CFU, colony-forming unit; GI, gastrointestinal; DM, diabetes mellitus; HOMA-IR, homeostasis model assessment of insulin resistance; CRP, C-reactive protein; HDL, high-density lipoprotein; KT, kidney transplantation. 
composition of the microbiota, AST-120 decreased serum IS levels, reduced the production of ROS by endothelial cells, and impeded subsequent oxidative stress and inflammation [79,80]. Several clinical studies have also shown that AST-120 reduced uremic symptoms, proteinuria and time-to-dialysis in CKD patients [79,81]. However, a recent meta-analysis reported that AST-120 reduced serum IS levels but showed no significant improvement of renal function and all-cause mortality [82]. Sevelamer (hydrochloride or carbonate), a polyphosphate binder, is noted for its ability to bind uremic toxins. Although some experiments and/or clinical studies report beneficial effects on reducing uremic toxins, such as serum IS and pCS levels, the results of the clinical studies published to date are conflicting [83].

\section{CONCLUSIONS}

The microenvironmental interface between the gut microbiota and kidney is representative of the relationship that maintains the metabolic and immunologic milieu in humans. Uremic toxins, LPSs as endotoxins, disrupted intestinal barriers, and immune dysregulation are responsible for the bidirectional imbalance in which CKD predisposes to an alteration of gut microbial elements, and the resulting gut dysbiosis induces stage progression and the complications of CKD and, consequently, the effect of being trapped in a reciprocally vicious cycle. Clinical interventions designed to restore the imbalance of gut-kidney symbiosis have emerged as possible treatment options such as acceptable diets including high fiber meals, prebiotic, probiotic, and synbiotic supplementations, adsorbent agents, and FMT. Further clinical studies are required to confirm the safety and efficacy of these novel approaches to the improvement of disease outcomes and survival of patients with CKD.

\section{Conflict of interest}

No potential conflict of interest relevant to this article was reported.

\section{Acknowledgments}

This study was financially supported by the research fund of Dankook University in 2019.

\section{REFERENCES}

1. Chen TK, Knicely DH, Grams ME. Chronic kidney disease diagnosis and management: a review. JAMA 2019;322:12941304.

2. Kim KM, Oh HJ, Choi HY, Lee H, Ryu DR. Impact of chronic kidney disease on mortality: a nationwide cohort study. Kidney Res Clin Pract 2019;38:382-390.

3. Jin DC. Analysis of mortality risk from Korean hemodialysis registry data 2017. Kidney Res Clin Pract 2019;38:169-175.

4. Rooks MG, Garrett WS. Gut microbiota, metabolites and host immunity. Nat Rev Immunol 2016;16:341-352.

5. Lin L, Zhang J. Role of intestinal microbiota and metabolites on gut homeostasis and human diseases. BMC Immunol 2017;18:2.

6. Kanbay M, Onal EM, Afsar B, et al. The crosstalk of gut microbiota and chronic kidney disease: role of inflammation, proteinuria, hypertension, and diabetes mellitus. Int Urol Nephrol 2018;50:1453-1466.

7. Yang T, Richards EM, Pepine CJ, Raizada MK. The gut microbiota and the brain-gut-kidney axis in hypertension and chronic kidney disease. Nat Rev Nephrol 2018;14:442456.

8. Plata C, Cruz C, Cervantes LG, Ramirez V. The gut microbiota and its relationship with chronic kidney disease. Int Urol Nephrol 2019;51:2209-2226.

9. Mahmoodpoor F, Rahbar Saadat Y, Barzegari A, Ardalan M, Zununi Vahed S. The impact of gut microbiota on kidney function and pathogenesis. Biomed Pharmacother 2017;93:412-419.

10. Meijers B, Evenepoel P, Anders HJ. Intestinal microbiome and fitness in kidney disease. Nat Rev Nephrol 2019;15:531545 .

11. Kang JY. The gastrointestinal tract in uremia. Dig Dis Sci 1993;38:257-268.

12. Lim E, Hyun S, Lee JM, et al. Effects of education on low-phosphate diet and phosphate binder intake to control serum phosphate among maintenance hemodialysis patients: a randomized controlled trial. Kidney Res Clin Pract 2018;37:69-76.

13. Yang J, Lim SY, Ko YS, et al. Intestinal barrier disruption and dysregulated mucosal immunity contribute to kidney fibrosis in chronic kidney disease. Nephrol Dial Transplant 2019;34:419-428.

14. De Almeida Duarte JB, de Aguilar-Nascimento JE, Nascimento M, Nochi RJ Jr. Bacterial translocation in experi- 
mental uremia. Urol Res 2004;32:266-270.

15. Yun $\mathrm{Y}$, Yin $\mathrm{H}, \mathrm{Gao} \mathrm{Z}$, et al. Intestinal tract is an important organ for lowering serum uric acid in rats. PLoS One 2017;12:e0190194.

16. Vaziri ND, Wong J, Pahl M, et al. Chronic kidney disease alters intestinal microbial flora. Kidney Int 2013;83:308-315.

17. Wong J, Piceno YM, DeSantis TZ, Pahl M, Andersen GL, Vaziri ND. Expansion of urease- and uricase-containing, indole- and p-cresol-forming and contraction of shortchain fatty acid-producing intestinal microbiota in ESRD. Am J Nephrol 2014;39:230-237.

18. Farhadi A, Banan A, Fields J, Keshavarzian A. Intestinal barrier: an interface between health and disease. J Gastroenterol Hepatol 2003;18:479-497.

19. Steinberg D, Bennett GG, Svetkey L. The DASH diet, 20 years later. JAMA 2017;317:1529-1530.

20. Chiavaroli L, Mirrahimi A, Sievenpiper JL, Jenkins DJ, Darling PB. Dietary fiber effects in chronic kidney disease: a systematic review and meta-analysis of controlled feeding trials. Eur J Clin Nutr 2015;69:761-768.

21. Lu L, Huang YF, Wang MQ, et al. Dietary fiber intake is associated with chronic kidney disease (CKD) progression and cardiovascular risk, but not protein nutritional status, in adults with CKD. Asia Pac J Clin Nutr 2017;26:598-605.

22. Koppe L, Fouque D, Soulage CO. The role of gut microbiota and diet on uremic retention solutes production in the context of chronic kidney disease. Toxins (Basel) 2018;10:155.

23. Rukavina Mikusic NL, Kouyoumdzian NM, Choi MR. Gut microbiota and chronic kidney disease: evidences and mechanisms that mediate a new communication in the gastrointestinal-renal axis. Pflugers Arch 2020;472:303-320.

24. Wu IW, Hsu KH, Lee CC, et al. p-Cresyl sulphate and indoxyl sulphate predict progression of chronic kidney disease. Nephrol Dial Transplant 2011;26:938-947.

25. Miyazaki T, Ise M, Seo H, Niwa T. Indoxyl sulfate increases the gene expressions of TGF-beta 1, TIMP-1 and pro-alpha 1 (I) collagen in uremic rat kidneys. Kidney Int Suppl 1997;62:S15-S22.

26. Ichii O, Otsuka-Kanazawa S, Nakamura T, et al. Podocyte injury caused by indoxyl sulfate, a uremic toxin and aryl-hydrocarbon receptor ligand. PLoS One 2014;9:e108448.

27. Han H, Zhu J, Zhu Z, et al. p-Cresyl sulfate aggravates cardiac dysfunction associated with chronic kidney disease by enhancing apoptosis of cardiomyocytes. J Am Heart Assoc 2015;4:e001852.

28. Sun CY, Chang SC, Wu MS. Uremic toxins induce kidney fibrosis by activating intrarenal renin-angiotensin-aldosterone system associated epithelial-to-mesenchymal transition. PLoS One 2012;7:e34026.

29. Pelletier CC, Croyal M, Ene L, et al. Elevation of trimethylamine-N-oxide in chronic kidney disease: contribution of decreased glomerular filtration rate. Toxins (Basel) 2019;11:635.

30. Tang WH, Wang Z, Kennedy DJ, et al. Gut microbiota-dependent trimethylamine $\mathrm{N}$-oxide (TMAO) pathway contributes to both development of renal insufficiency and mortality risk in chronic kidney disease. Circ Res 2015;116:448-455.

31. Stubbs JR, House JA, Ocque AJ, et al. Serum trimethylamine-N-oxide is elevated in CKD and correlates with coronary atherosclerosis burden. J Am Soc Nephrol 2016;27:305-313.

32. Szeto CC, Kwan BC, Chow KM, et al. Endotoxemia is related to systemic inflammation and atherosclerosis in peritoneal dialysis patients. Clin J Am Soc Nephrol 2008;3:431436.

33. Ramezani A, Raj DS. The gut microbiome, kidney disease, and targeted interventions. J Am Soc Nephrol 2014;25:657670.

34. Neal MD, Leaphart C, Levy R, et al. Enterocyte TLR4 mediates phagocytosis and translocation of bacteria across the intestinal barrier. J Immunol 2006;176:3070-3079.

35. Rice JB, Stoll LL, Li WG, et al. Low-level endotoxin induces potent inflammatory activation of human blood vessels: inhibition by statins. Arterioscler Thromb Vasc Biol 2003;23:1576-1582.

36. McIntyre CW, Harrison LE, Eldehni MT, et al. Circulating endotoxemia: a novel factor in systemic inflammation and cardiovascular disease in chronic kidney disease. Clin J Am Soc Nephrol 2011;6:133-141.

37. Jazani NH, Savoj J, Lustgarten M, Lau WL, Vaziri ND. Impact of gut dysbiosis on neurohormonal pathways in chronic kidney disease. Diseases 2019;7:21.

38. Onal EM, Afsar B, Covic A, Vaziri ND, Kanbay M. Gut microbiota and inflammation in chronic kidney disease and their roles in the development of cardiovascular disease. Hypertens Res 2019;42:123-140.

39. Cosola C, Rocchetti MT, Sabatino A, Fiaccadori E, Di Iorio BR, Gesualdo L. Microbiota issue in CKD: how promising are gut-targeted approaches? J Nephrol 2019;32:27-37. 
40. Baek SH, Cha RH, Kang SW, et al. Circulating renalase predicts all-cause mortality and renal outcomes in patients with advanced chronic kidney disease. Korean J Intern Med 2019;34:858-866.

41. Park GH, Song JW, Lee CM, et al. Long-term prognosis of end-stage renal disease patients with normal myocardial perfusion as determined by single photon emission computed tomography. Korean J Intern Med 2018;33:148-156.

42. Lin CJ, Wu V, Wu PC, Wu CJ. Meta-analysis of the associations of p-cresyl sulfate (PCS) and indoxyl sulfate (IS) with cardiovascular events and all-cause mortality in patients with chronic renal failure. PLoS One 2015;10:e0132589.

43. Heianza Y, Ma W, Manson JE, Rexrode KM, Qi L. Gut microbiota metabolites and risk of major adverse cardiovascular disease events and death: a systematic review and meta-analysis of prospective studies. J Am Heart Assoc 2017;6:e004947.

44. Sohn BK, Oh YK, Choi JS, et al. Effectiveness of group cognitive behavioral therapy with mindfulness in end-stage renal disease hemodialysis patients. Kidney Res Clin Pract 2018;37:77-84.

45. Agusti A, Garcia-Pardo MP, Lopez-Almela I, et al. Interplay between the gut-brain axis, obesity and cognitive function. Front Neurosci 2018;12:155.

46. Ohtsuki S, Asaba H, Takanaga $\mathrm{H}$, et al. Role of blood-brain barrier organic anion transporter $3\left(\mathrm{OAT}_{3}\right)$ in the efflux of indoxyl sulfate, a uremic toxin: its involvement in neurotransmitter metabolite clearance from the brain. J Neurochem 2002;83:57-66.

47. Lin YT, Wu PH, Liang SS, et al. Protein-bound uremic toxins are associated with cognitive function among patients undergoing maintenance hemodialysis. Sci Rep 2019;9:20388.

48. Watanabe I, Tatebe J, Namba S, Koizumi M, Yamazaki J, Morita T. Activation of aryl hydrocarbon receptor mediates indoxyl sulfate-induced monocyte chemoattractant protein-1 expression in human umbilical vein endothelial cells. Circ J 2013;77:224-230.

49. Kwon YE, Choi HY, Kim S, Ryu DR, Oh HJ; ESRD Registry Committee of the Korean Society of Nephrology. Fracture risk in chronic kidney disease: a Korean population-based cohort study. Kidney Res Clin Pract 2019;38:220-228.

50. Mozar A, Louvet L, Godin C, et al. Indoxyl sulphate inhibits osteoclast differentiation and function. Nephrol Dial Transplant 2012;27:2176-2181.

51. Park JS, Choi HI, Bae EH, Ma SK, Kim SW. Paricalcitol attenuates indoxyl sulfate-induced apoptosis through the inhibition of MAPK, Akt, and NF-kB activation in HK-2 cells. Korean J Intern Med 2019;34:146-155.

52. Nii-Kono T, Iwasaki Y, Uchida M, et al. Indoxyl sulfate induces skeletal resistance to parathyroid hormone in cultured osteoblastic cells. Kidney Int 2007;71:738-743.

53. Goto S, Fujii H, Hamada Y, Yoshiya K, Fukagawa M. Association between indoxyl sulfate and skeletal resistance in hemodialysis patients. Ther Apher Dial 2010;14:417-423.

54. Kalmokoff M, Zwicker B, O'Hara M, et al. Temporal change in the gut community of rats fed high amylose cornstarch is driven by endogenous urea rather than strictly on carbohydrate availability. J Appl Microbiol 2013;114:1516-1528.

55. Kieffer DA, Piccolo BD, Vaziri ND, et al. Resistant starch alters gut microbiome and metabolomic profiles concurrent with amelioration of chronic kidney disease in rats. Am J Physiol Renal Physiol 2016;310:F857-F871.

56. Vaziri ND, Liu SM, Lau WL, et al. High amylose resistant starch diet ameliorates oxidative stress, inflammation, and progression of chronic kidney disease. PLoS One 2014;9:e114881.

57. Krishnamurthy VM, Wei G, Baird BC, et al. High dietary fiber intake is associated with decreased inflammation and all-cause mortality in patients with chronic kidney disease. Kidney Int 2012;81:300-306.

58. Gibson GR, Roberfroid MB. Dietary modulation of the human colonic microbiota: introducing the concept of prebiotics. J Nutr 1995;125:1401-1412.

59. Gibson GR, Probert HM, Loo JV, Rastall RA, Roberfroid MB. Dietary modulation of the human colonic microbiota: updating the concept of prebiotics. Nutr Res Rev 2004;17:259-275.

6o. Meijers BK, De Preter V, Verbeke K, Vanrenterghem Y, Evenepoel P. p-Cresyl sulfate serum concentrations in haemodialysis patients are reduced by the prebiotic oligofructose-enriched inulin. Nephrol Dial Transplant 2010;25:219224.

61. Sueyoshi M, Fukunaga M, Mei M, et al. Effects of lactulose on renal function and gut microbiota in adenine-induced chronic kidney disease rats. Clin Exp Nephrol 2019;23:908919.

62. Rastall RA, Gibson GR, Gill HS, et al. Modulation of the microbial ecology of the human colon by probiotics, prebiotics and synbiotics to enhance human health: an overview of enabling science and potential applications. FEMS Microbiol Ecol 2005;52:145-152. 
63. Ranganathan N, Ranganathan P, Friedman EA, et al. Pilot study of probiotic dietary supplementation for promoting healthy kidney function in patients with chronic kidney disease. Adv Ther 2010;27:634-647.

64. Ranganathan N, Friedman EA, Tam P, Rao V, Ranganathan $\mathrm{P}$, Dheer R. Probiotic dietary supplementation in patients with stage 3 and 4 chronic kidney disease: a 6-month pilot scale trial in Canada. Curr Med Res Opin 2009;25:19191930.

65. Guida B, Germano R, Trio R, et al. Effect of short-term synbiotic treatment on plasma p-cresol levels in patients with chronic renal failure: a randomized clinical trial. Nutr Metab Cardiovasc Dis 2014;24:1043-1049.

66. Viramontes-Horner D, Marquez-Sandoval F, Martin-del-Campo F, et al. Effect of a symbiotic gel (Lactobacillus acidophilus + Bifidobacterium lactis + inulin) on presence and severity of gastrointestinal symptoms in hemodialysis patients. J Ren Nutr 2015;25:284-291.

67. Esgalhado M, Kemp JA, Azevedo R, et al. Could resistant starch supplementation improve inflammatory and oxidative stress biomarkers and uremic toxins levels in hemodialysis patients?: a pilot randomized controlled trial. Food Funct 2018;9:6508-6516.

68. Poesen R, Evenepoel P, de Loor H, et al. The influence of prebiotic arabinoxylan oligosaccharides on microbiota derived uremic retention solutes in patients with chronic kidney disease: a randomized controlled trial. PLoS One 2016;11:e0153893.

69. Ramos CI, Armani RG, Canziani ME, et al. Effect of prebiotic (fructooligosaccharide) on uremic toxins of chronic kidney disease patients: a randomized controlled trial. Nephrol Dial Transplant 2019;34:1876-1884.

70. Laffin MR, Tayebi Khosroshahi H, Park H, et al. Amylose resistant starch (HAM-RS2) supplementation increases the proportion of Faecalibacterium bacteria in end-stage renal disease patients: microbial analysis from a randomized placebo-controlled trial. Hemodial Int 2019;23:343-347.

71. Eidi F, Poor-Reza Gholi F, Ostadrahimi A, Dalili N, Samadian F, Barzegari A. Effect of Lactobacillus rhamnosus on serum uremic toxins (phenol and p-cresol) in hemodialysis patients: a double blind randomized clinical trial. Clin Nutr ESPEN 2018;28:158-164.

72. Mafi A, Namazi G, Soleimani A, Bahmani F, Aghadavod E, Asemi Z. Metabolic and genetic response to probiotics supplementation in patients with diabetic nephropathy: a randomized, double-blind, placebo-controlled trial. Food Funct 2018;9:4763-4770.

73. Borges NA, Stenvinkel P, Bergman P, et al. Effects of probiotic supplementation on trimethylamine-N-oxide plasma levels in hemodialysis patients: a pilot study. Probiotics Antimicrob Proteins 2019;11:648-654.

74. Rossi M, Johnson DW, Morrison M, et al. Synbiotics Easing Renal Failure by Improving Gut Microbiology (SYNERGY): a randomized trial. Clin J Am Soc Nephrol 2016;11:223-231.

75. Guida B, Cataldi M, Memoli A, et al. Effect of a shortcourse treatment with synbiotics on plasma p-cresol concentration in kidney transplant recipients. J Am Coll Nutr 2017;36:586-591.

76. Dehghani H, Heidari F, Mozaffari-Khosravi H, Nouri-Majelan N, Dehghani A. Synbiotic supplementations for azotemia in patients with chronic kidney disease: a randomized controlled trial. Iran J Kidney Dis 2016;10:351-357.

77. Thongprayoon C, Cheungpasitporn W, Phatharacharukul P, Mahaparn P, Bruminhent J. High mortality risk in chronic kidney disease and end stage kidney disease patients with clostridium difficile infection: a systematic review and meta-analysis. J Nat Sci 2015;1:e85.

78. Kim SC, Seo MY, Lee JY, et al. Advanced chronic kidney disease: a strong risk factor for Clostridium difficile infection. Korean J Intern Med 2016;31:125-133.

79. Liu WC, Tomino Y, Lu KC. Impacts of indoxyl sulfate and p-cresol sulfate on chronic kidney disease and mitigating effects of AST-120. Toxins (Basel) 2018;10:367.

8o. Sato E, Hosomi K, Sekimoto A, et al. Effects of the oral adsorbent AST-12o on fecal p-cresol and indole levels and on the gut microbiota composition. Biochem Biophys Res Commun 2020;525:773-779.

81. Cha RH, Kang SW, Park CW, et al. Sustained uremic toxin control improves renal and cardiovascular outcomes in patients with advanced renal dysfunction: post-hoc analysis of the Kremezin Study against renal disease progression in Korea. Kidney Res Clin Pract 2017;36:68-78.

82. Chen YC, Wu MY, Hu PJ, et al. Effects and safety of an oral adsorbent on chronic kidney disease progression: a systematic review and meta-analysis. J Clin Med 2019;8:1718.

83. Rodriguez-Osorio L, Zambrano DP, Gracia-Iguacel C, et al. Use of sevelamer in chronic kidney disease: beyond phosphorus control. Nefrologia 2015;35:207-217. 